\title{
Traffic Monitoring using SRTM Along-Track Interferometry
}

\author{
H. Breit, M. Eineder, J. Holzner, H. Runge, R. Bamler \\ German Aerospace Center (DLR), Oberpfaffenhofen, D-82234 Wessling, Germany \\ Phone: +49-8153-28-1369 Fax: +49-8153-28-1444, e-mail: Helko.Breit@dlr.de
}

\begin{abstract}
In February 2000, the Shuttle Radar Topography Mission SRTM was flown as the first single-pass SAR interferometer in space. The goal was to obtain a global digital elevation model. Therefore, a secondary receive-only antenna on the tip of a $60 \mathrm{~m}$ long deployable mast supplemented the primary transmit and receive antenna mounted in the shuttle cargo bay. Due to mechanical constraints, the interferometric baseline did not only consist of the terrain height sensitive across track component but also contained an along track component of $7 \mathrm{~m}$. The resulting time lag between the data acquisitions by the two antennas causes phase differences, which are proportional to the line-ofsight velocity of moving targets. Since this system ability has been expected prior to the SRTM launch, a traffic monitoring experiment has been carried out during the mission, which showed a surprisingly good agreement between the GPS velocity measurements on ground and the measurement from space. A strategy to exploit the along track interferometric (ATI) phase and the coherence together with the azimuth displacement of moving vehicles in focused SAR images has been developed and applied on sample test sides for traffic monitoring on highways.
\end{abstract}

\section{INTRODUCTION}

For traffic flow monitoring and control the need for remote sensing data, acquired day and night under all weather conditions, becomes a demanding issue. Adequately designed SAR systems could be an answer [6]. This was the motivation for the experiment being presented.

\section{MOVING TARGETS IN THE SRTM DATA}

In the following we discuss special effects caused by moving targets. We assume the SRTM/X-SAR case and targets moving with a constant velocity. TABLE I. comprises important sensor parameters as well as the acquisition parameters of the moving target experiment.

TABLE I

\begin{tabular}{|l|l|}
\hline \multicolumn{2}{|c|}{ SRTM / X-SAR Sensor, Acquisition and Processing Parameters } \\
\hline wavelength $(\lambda)$ & $0.03123 \mathrm{~m}$ \\
\hline antenna length & $12 \mathrm{~m}$ \\
\hline orbit altitude & $233 \mathrm{~km}$ \\
\hline range bandwidth & $9.5 \mathrm{MHz}$ \\
\hline pulse repitition frequency $(\mathrm{PRF})$ & $1674 \mathrm{~Hz}$ \\
\hline target distance $\left(\mathrm{R}_{\text {target }}\right)$ & $403400 \mathrm{~m}$ \\
\hline incidence angle $\left(\theta_{\mathrm{i}}\right)$ & $53.65 \mathrm{deg}$. \\
\hline track angle difference $\left(\Delta \Psi_{\text {track }}\right)$ & $86.57 \mathrm{deg}$. \\
\hline
\end{tabular}

\begin{tabular}{|l|l|}
\hline \multicolumn{2}{|c|}{ SRTM / X-SAR Sensor, Acquisition and Processing Parameters } \\
\hline Doppler rate (FM) & $8647 \mathrm{~Hz} / \mathrm{sec}$ \\
\hline azimuth processing bandwidth & $1180 \mathrm{~Hz}$ \\
\hline processed aperture time & $0.14 \mathrm{sec}$ \\
\hline
\end{tabular}

\section{A. SAR Imaging Properties}

The radial component $\mathrm{v}_{\mathrm{r}}$ of the target velocity, relative to the line of sight, contributes an additional linear term to the target's phase history compared to the stationary case [2], [3]. Furthermore it adds to the range cell migration. In our case, a contribution of more than $8 \mathrm{~m}$, half of the range resolution cell, within the aperture time would require a target motion with a radial component of more than $\pm 200 \mathrm{~km} / \mathrm{h}$.

The tangential, along track, component $\mathrm{v}_{\mathrm{t}}$ of the target velocity alters the Doppler rate compared to the stationary case. Commonly agreed, for phase aberrations of $\pi / 4$ at both edges of the synthetic aperture, azimuth defocusing becomes significant. This limit is reached for Doppler rate mismatches higher than $1 /\left(4 \cdot\left(\mathrm{t}_{\text {aperture }} / 2\right)^{2}\right)=51 \mathrm{~Hz} / \mathrm{s}$. For a moving target located at near range $(\approx 390 \mathrm{~km})$ this would impose a tangential velocity higher than

$$
v_{\mathrm{t}}= \pm \sqrt{\left(\lambda \cdot R_{\text {target }}\right) /\left(8 \cdot t_{\text {aperture }}^{2}\right)} \approx \pm 2000 \mathrm{~km} / \mathrm{h} .
$$

Hence, in the velocity range considered here no defocusing due to additional range migration or due to a FM-rate mismatch will occur. In the following, only the radial component will be exploited for moving target indication (MTI) and velocity estimation.

\section{B. Azimuth Displacement Effect}

The Doppler shift caused by the additional linear phase term leads to an azimuth displacement of the focused target. The maximum unambiguous Doppler shift of $\pm \mathrm{PRF} / 2$ corresponds to a radial target motion of $\pm \lambda / 4$ (which results in a two-way distance of $\lambda / 2$ ) from pulse to pulse. Thus, without further knowledge, $v_{\mathrm{r}}$ may be unambiguously determined from azimuth displacement in the interval of $\pm \mathrm{PRF} \cdot \lambda / 4=$ $\pm 47.05 \mathrm{~km} / \mathrm{h}$. The displacement depends on the targets slant range $r$ :

$$
\Delta t_{\text {azimuth }}(r)=\frac{2 \cdot v_{r}}{\lambda \cdot F M(r)}
$$

Targets moving radially away from the sensor are displaced reverse to the flight direction.

\section{ATI Phase Effect}

The ATI phase [1], which computes to 


$$
\theta_{A T I}=(4 \pi / \lambda) \cdot \Delta t_{\text {acquisition }} \cdot v_{r},
$$

is unambiguously obtained, as long as the target moves radially less than $\pm \lambda / 4$ in the time between the two data acquisitions. For the time lag $\Delta \mathrm{t}_{\text {acquisition }}=0.00047 \mathrm{sec}, \mathrm{v}_{\mathrm{r}}$ can be unambiguously determined in the interval of $\pm 59.8 \mathrm{~km} / \mathrm{h}$, corresponding to $\theta_{\mathrm{ATI}}= \pm \pi$.

\section{Velocity Ambiguity Resolving}

Both velocity estimation methods are affected by wrapping and are therefore ambiguous. Since the wrapping velocity intervals for both methods are different, a two dimensional unwrapping approach can resolve the ambiguity for velocities which exceed the one dimensional maximum unambiguous velocity.

\section{EXPERIMENT}

\section{A. Test Target and Data Acquisition}

On February $18^{\text {th }}, 2000$ at 10:22 AM the area around Oberpfaffenhofen was interferometrically mapped by the Cand X-band SAR interferometers on board the shuttle on its $109^{\text {th }}$ orbit. Simultaneously, a car, equipped with a GPS receiver was driven on a road oriented in across track direction. Since the radar cross section of a passenger car contributes only by a small amount to the backscatter energy of the Xband resolution cell of about $120 \mathrm{~m}^{2}$, a Luneberg lens was mounted on the roof of the car in order to enhance its radar visibility.

\section{B. Data Processing}

Data processing has been accomplished by the operational SRTM/X-SAR InSAR processing chain, consisting of the DLR developed Chirp Scaling SAR processor BSAR and the interferometric processing system GENESIS [5], respectively. During SAR focusing the operationally applied azimuth filter bandwidth of $1180 \mathrm{~Hz}$ and spectral Hamming weighting of 0.75 have been maintained. The high Doppler shift of $-690 \mathrm{~Hz}$ for the moving car was not accounted for.

Figure 1. shows a simulated comparison of the azimuth compression results for a moving (red) and a stationary (black) target under the experiments acquisition conditions. The azimuth displacement of -134 samples and thus the radial velocity is close to the unambiguous measurement limits and the correlation peak power has dropped by about $8 \mathrm{~dB}$ while the energy is shifted into the azimuth ambiguity at azimuth sample $190(=-134+324)$.

Figure 2. shows a simulation of the correlation peak power loss as a function of the radial velocity, normalized to its unambiguous maximum. In a more complex implementation the Doppler centroid shift of the moving objects can be accounted for in the processing chain.

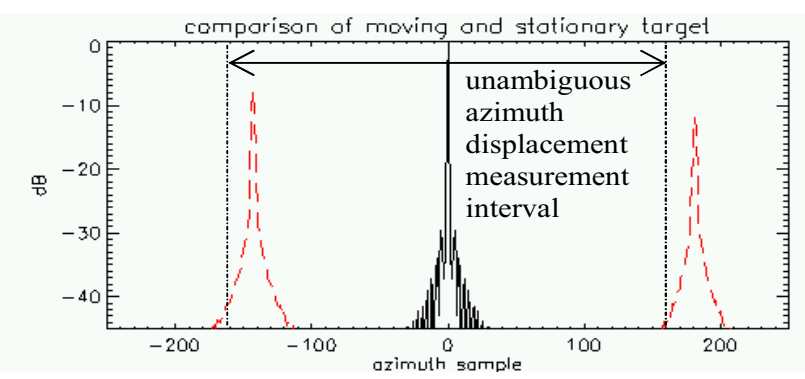

Figure 1. One-dimensional azimuth compression simulation for a stationary target and a moving target.

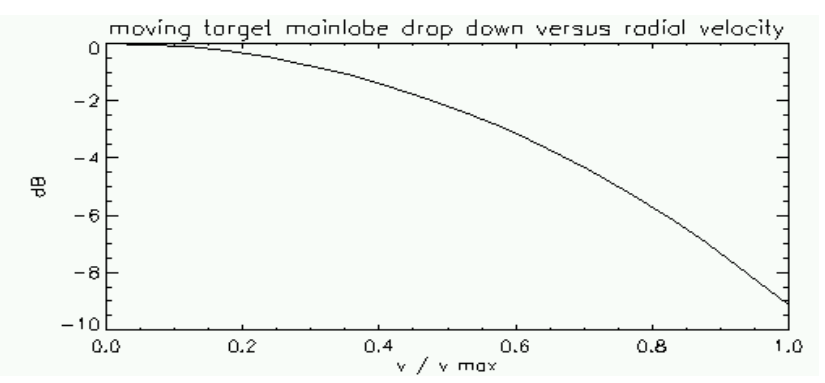

Figure 2. Loss of the correlation peak power of a focused moving target as a function of the normalized radial velocity.

\section{Data Evaluation and Results}

The coherence map, a by-product of interferometric SAR processing, is a valuable data set to detect moving targets. Therefore the one second time tick GPS measured WGS-84 car coordinates are projected into the SAR coordinates $(t, r)$, i.e. the zero-Doppler plane. From the neighboring one second time tick GPS measurements the exact car location at the time of SAR illumination is interpolated and the car heading angle $\psi_{\text {car }}$ is determined. Together with the incidence angle $\theta_{\mathrm{i}}$ and the satellite heading angle $\psi_{\text {satellite }}$ the car's radial velocity $v_{\mathrm{r}}$ computes to

$$
v_{r}=|v| \cdot \sin \left(\theta_{i}\right) \cdot \sin \left(\psi_{\text {satellite }}-\psi_{\text {car }}\right)=0.804 \cdot|v|
$$

Now the coherence loss caused by the car's radial motion induced ATI phase disturbance should be detectable within the azimuth ambiguity interval. The interval is $\left(\mathrm{PRF}^{2} / \mathrm{FM}(\mathrm{r})\right)$ $=324$ samples wide and centered at the target's zero Doppler position.

Finally the car's location is determined within the interpolated complex SAR image with sub pixel accuracy even if pixel level accuracy is sufficient due to the high sensitivity of the displacement effect. The phase is measured in the interferogram. Since the SRTM interferograms are dominated by the topographic phase, the terrain height contribution to the target's phase is estimated by averaging the phase of the surrounding samples.

TABLE II. summarizes the results and highlights the perfect agreement of the GPS measurements on ground and the velocity estimate derived from the azimuth displacement. 


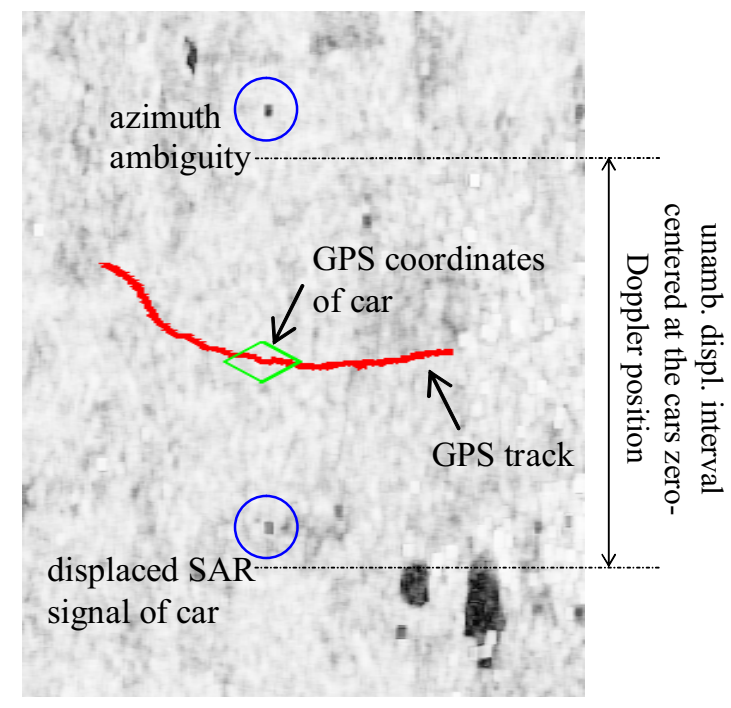

Figure 3. Coherence loss indicates the presence of a moving target. The larger low coherent areas are lakes.

TABLE II. RESULTS

\begin{tabular}{|l|l|}
\hline \multicolumn{2}{|c|}{ InSAR data evaluation } \\
\hline measured azimuth displacement & $130.6 \mathrm{pixel}$ \\
\hline radial velocity from displacement & $37.9 \mathrm{~km} / \mathrm{h}$ \\
\hline ground velocity from displacement & $\mathbf{4 7 . 1} \mathbf{~ k m} / \mathbf{h}$ \\
\hline ATI phase & $127 \mathrm{deg}$. \\
\hline radial velocity from ATI phase & $42.2 \mathrm{~km} / \mathrm{h}$ \\
\hline ground velocity from ATI phase & $52.5 \mathrm{~km} / \mathrm{h}$ \\
\hline \multicolumn{2}{|c|}{ GPS measurement } \\
\hline ground velocity & $\mathbf{4 8 . 3 3} \mathbf{~ k m} / \mathbf{h}$ \\
\hline
\end{tabular}

\section{SUMMARY AND OUTLOOK}

It is shown that the velocity of a car has been exactly measured from space even without the application of specialized MTI SAR processing algorithms. The coherence map of the ATI phase allows reasonable moving target identification. Additionally the ATI phase itself enables a two dimensional resolving of the velocity ambiguity. The azimuth displacement, which is exploited for the precise determination of the radial velocity, is measured as the distance between the misplaced signal occurrence in the SAR data and the GPS derived zero-Doppler position. Thus, the use of a geographical information system (GIS) seams to be indispensable in order to precisely determine the azimuth displacement from the highways and in order to determine the highway orientations, i.e. the car track angle $\psi_{\text {car }}$. Figure 4 . shows that trucks which are misplaced along both sides of the motorway can be identified in the coherence image. The upcoming TerraSAR-X mis- sion will be predestined for traffic monitoring applications due to the sensor's extremely high resolution imaging capabilities in combination with its ATI mode, enabled by the optional dual receive antenna configuration [6].

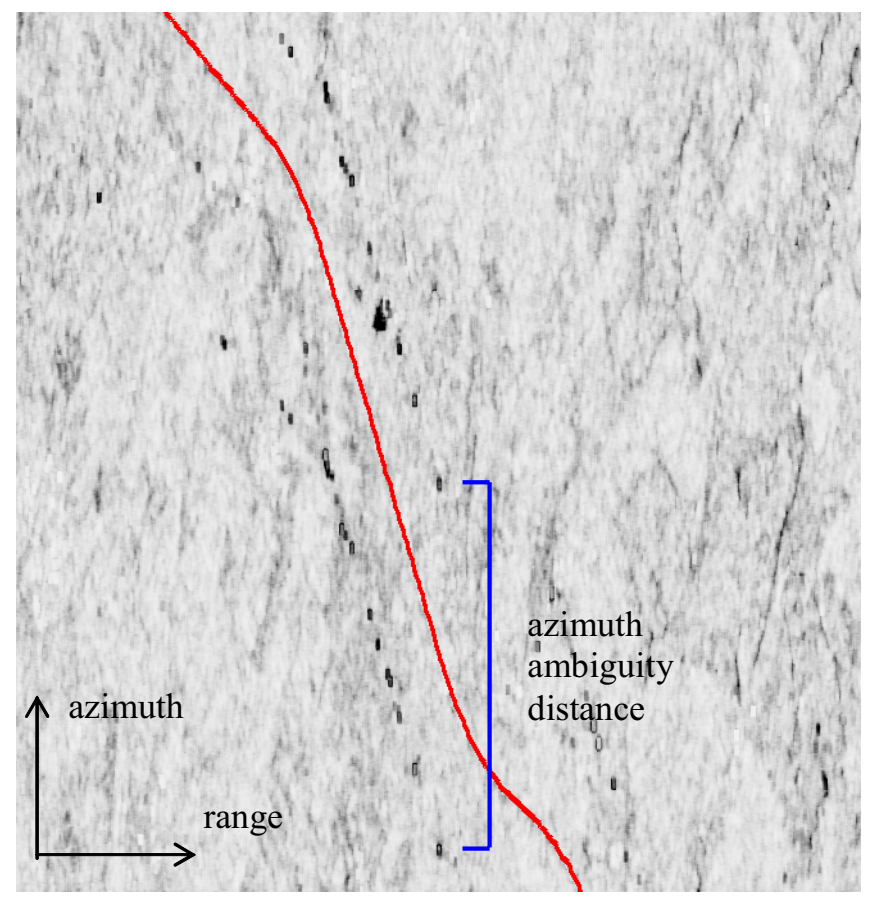

Figure 4. Coherence map featuring a $3 \mathrm{~km}$ long section of the Autobahn Munich - Nuernberg.

\section{ACKNOWLEDGMENT}

The authors would like to thank Joao Moreira and Markus Schwäbisch for the fruitful discussions about the subject and Manfred Zink, who proposed to use the Luneberg lens.

\section{REFERENCES}

[1] R. Bamler and P. Hartl, "Synthetic aperture radar interferometry", Inverse Problems 14, 1998.

[2] J. R. Moreira and W. Keydel, "A new MTI -SAR approach using the reflectivity displacement method", IEEE Transactions on Geoscience and Remote Sensing., vol. 33, no. 5, pp. 1238-1244, 1995.

[3] J. K. Jao, "Theory of Synthetic Aperture Radar Imaging of a Moving Target”, IEEE Trans. Geosci. Remote Sensing, vol. 39, no. 9, pp. $1984-$ $1992,2001$.

[4] A. A. Thompson and C. E. Livingstone, "Moving target performance for RADARSAT-2", IGARSS 2000, Honolulu.

[5] N. Adam, M. Eineder, H. Breit, S. Suchandt, "Shuttle Radar Topography Mission (SRTM): DLR's Interferometric SAR Processor for the Generation of a Global Digital Elevation Model", Sec. Int. Workshop on ERS SAR Interferometry FRINGE'99, Liège, Belgium, 1999.

[6] J. Mittermayer and H. Runge, "Conceptual Studies for exploiting the TerraSAR-X dual receive antenna", IGARSS 2003, Toulouse.

[7] R. Romeiser, H. Breit, M. Eineder, H. Runge, "Demonstration of current measurements from space by along-track SAR interferometry with SRTM data", IGARSS 2002, Toronto. 